\title{
Prime time for a polypill after myocardial infarction?
}

\author{
Valentin Fuster
}

Cardiovascular disease is the leading cause of death in developed, as well as developing, countries. Patients with key risk factors, and those who have a history of acute myocardial infarction (MI), are at high risk of coronary events. The efficacy of secondary cardiovascular prevention therapy in these populations is well documented, but is hampered by limited availability and inadequate prescription of medication, poor adherence to treatment, limited availability of medications, and unaffordable treatment costs. The use of fixed-dose combination drugs could circumvent these problems; however, controversy about the true value of a cardiovascular 'polypill' has been rife since 2003 when Wald and Law originally proposed the idea (Wald NJ and Law MR [2003] BMJ 326: 1419). Four articles in this issue of Nature Clinical Practice Cardiovascular Medicine readdress the concept of a cardiovascular polypill and provide new light for the immediate future of this controversial therapy.

In his Viewpoint article, Lennart Forslund, from the Medical Products Agency in Uppsala, Sweden, points out that the implementation of a polypill requires a new way of thinking on aspects such as treatment paradigms, drug development, and study design. Such changes in thinking will require interactions, not only between sponsors and regulatory agencies, but also the between the scientific community, health-care systems and political systems as a whole.

A Review by Drs Guglietta and Guerrero from Ferrer Laboratories, Barcelona, Spain address the unique challenges in the pharmaceutical development of a cardiovascular polypill, such as the one they have developed for Centro Nacional de Investigaciones Cardiovasculares. The final decision on which, and how many, active drugs should be included in the Polypill depends on how developers prioritize clinical, pharmaceutical, and commercial issues.

In our Review, Ginés Sanz and I propose a three-component polypill, comprising aspirin, "...controversy about the true value of a cardiovascular 'Polypill' has been rife since 2003 when Wald and Law originally proposed the idea."

$V$ Fuster is the Editorin-Chief of Nature Clinical Practice Cardiovascular Medicine.

\section{Competing interests}

The author declared no competing interests.

www.nature.com/clinicalpractice doi:10.1038/ncpcardio1443 a statin, and an angiotensin-convertingenzyme inhibitor for secondary prevention in patients who have already suffered an acute MI. The product is in the final stages of development and will be made available at a price that will allow this Polypill to be accessible to patients in low-income countries. Although Wald and Law originally proposed a pill combining half-doses of three antihypertensive drugs together with a statin, folic acid, and aspirin for primary prevention, our Polypill will include $100 \mathrm{mg}$ aspirin, $40 \mathrm{mg}$ simvastatin, and ramipril at three different doses $(2.5 \mathrm{mg}$, $5 \mathrm{mg}$, and $10 \mathrm{mg}$ ), to facilitate dose titration. Our fixed-dose combination therapy will be targeted towards high-risk patients who have had an Ml and who should already have received these agents, as recommended by various guidelines. Although $\beta$-blockers should be routinely administered in patients following MI, we decided do not include them in our Polypill, as the addition of a $\beta$-blocker would increase the difficulties in galaenic formulation and bioequivalence. Furthermore, a four-component combined therapy would narrow the target population and increase the difficultly of dose titration.

Also in this issue of the journal, Salim Yusuf and colleagues describe the Indian Polycap Study, which comprehensively tests Wald and Law's hypothesis with a five-drug combination as part of a multicenter, randomized, controlled, double-blind trial in a primary prevention setting. The 'Polycap' contains atenolol, thiazide, ramipril, simvastatin, and aspirin. Many will argue that this combination of agents is unnecessary for primary cardiovascular prevention. A fixed-dose polypill for use by patients with $\mathrm{Ml}$ is, however, less controversial because each component is required for treatment of these individuals, adherence to therapy could be improved, and the cost of treatment could be reduced, thus making therapy more affordable in low-income countries. 